\title{
REPRESENTAÇÕES NO EMPIRISMO CONCEITUAL DE PRINZ
}

\section{REPRESENTATIONS IN PRINZ'S CONCEPTUAL EMPIRICISM}

\author{
César Fernando Meurer*
}

Recebido: 03/2017

Aprovado: 09/2017

\begin{abstract}
Resumo: A proxytype theory de Jesse Prinz vincula, mas não identifica, representações e conceitos. Ofereço uma apresentação geral dessa teoria, tratando de realçar que conceitos são entendidos como cópias de representações perceptuais. Em seguida, apresento e critico a concepção de intencionaldade que Prinz subscreve. Com efeito, penso que essa teoria da intencionalidade é imprecisa no que tange à determinação da referência.

Palavras-chave: Representações perceptuais; Conceitos; Intencionalidade.
\end{abstract}

\begin{abstract}
Jesse Prinz's proxytype theory links, but does not identify, representations and concepts. I offer a general presentation of this theory, highlighting that concepts are understood as copies of perceptual representations. Afterwards, I lay out and criticize Prinz's conception of intentionality. Indeed, I think it is imprecise in determining reference.

Keywords: Perceptual representations; Concepts; Intentionality.
\end{abstract}

O que são conceitos? O que é possuir um conceito? $O$ que são representações? Nossa mente opera com representações? Possuir uma representação é o mesmo que possuir um conceito? Se não, como distinguir representações de conceitos?

Essas perguntas, e inúmeras outras que se desdobram delas, já foram respondidas de diversas maneiras por filósofos, psicólogos e estudiosos de outras áreas. Vincular conceitos a representações é uma opção teórica, por assim dizer. Alternativamente, conceitos podem ser vinculados com habilidades (na linha do segundo Wittgenstein) ou com objetos abstratos (na linha de Frege). De qualquer modo, o tema não se deixa delimitar facilmente: estão em jogo suposições ontológicas, epistemológicas e semânticas. Por conta disso, disputas nesse terreno "often reflect deeply opposing approaches to the study of the mind, to language, and even to philosophy it self" (Margolis e Laurence, 2012, s/p).

O presente trabalho aborda alguns aspectos da teoria de conceitos proposta por Jesse Prinz: a proxytype theory. Trata-se de uma teoria de matriz empirista, que veio a lume em Furnishing the mind (2002) e foi retomada em The return of concept empiricism (2005). Meu primeiro objetivo é oferecer uma visão geral dessa teoria. Para tanto, sublinharei algumas ideias que considero basilares para compreender como Prinz vincula, mas não identifica, representações e conceitos. O segundo intento é expor o tratamento que a proxytype theory confere ao conteúdo intencional. Prinz subscreve uma teoria informacional: um conceito refere aquilo que tem o poder de causá-lo. Por fim, ensaiarei um contraexemplo para essa concepção de intencionalidade. Se o meu contraexemplo for razoável, então a proxytype theory é imprecisa no que

\footnotetext{
* Doutor em filosofia. Pesquisador do Social-Brains Research Group/Unisinos. Professor da Unilasalle Canoas. 
tange à determinação da referência.

\section{A proxytype theory: notas gerais}

Nesta seção, ofereço uma visão geral do empirismo conceitual de Prinz. Deve ficar claro como ele vincula conceitos e representações. Vincular, já mencionei na introdução, não significa identificar. Com efeito, afasto-me da interpretação que considera que a "proxytype theory identifies concepts with long-term memory networks of perceptual representations [...]" (Rosa, 2005, p. 597 - itálico acrescentado).

Furnishing the mind (2002) é oriundo do trabalho com o qual Prinz obteve o título de doutor em filosofia da Universidade de Chicago, em 1997. O título, aliás, ecoa a principal questão que Locke procurou responder no An essay concerning human understanding: "Let us then suppose the mind to be, as we say, white paper, void of all characters, without any ideas: How comes it to be furnished? [...] Whence has it all the materials of reason and knowledge? (Locke, Essay, Book II, Ch.1, §2, p. 51 - itálicos acrescentados).

Locke não falava em conceitos, mas em ideias. Para ele, toda e qualquer ideia é produto da experiência; possui bases perceptuais. No Essay lemos que a mente obtém sua "matéria-prima" dos sentidos. Os estados mentais que acompanham nossas experiências servem para "mobiliar" a mente, isto é, para capacitar o pensamento, o planejamento, a solução de problemas. Essa visão ficou conhecida como 'empirismo'.

Prinz assume o legado empirista no que se refere à natureza dos conceitos: "All (human) concepts are copies or combinations of copies of perceptual representations" (2002, p. 108). Como interpretar essa afirmação? O que são representações perceptuais? O que significa dizer que conceitos são cópias de representações? A cópia de uma representação é também uma representação? Vejamos.

\section{Representações perceptuais}

Recebem o adjetivo 'perceptuais' aquelas representações que possuem origem nos sentidos. Isso parece trivial, mas não é. A justa compreensão da proxytype theory demanda não confundir representações perceptuais com conceitos. Como vimos, estes são cópias daquelas. De acordo com Prinz, "the most direct path to distinguishing perceptual representations from concepts is not to isolate privileged semantic properties but to distinguish the senses from other cognitive faculties" (Prinz, 2002, p. 113). Quer dizer: para aclarar o que 
são representações perceptuais precisamos explicar o que são os sentidos ou, como alguns preferem, o sistema sensório.

O que é essa faculdade que chamamos 'sentidos' ou 'sistema sensório'? Possui o sistema sensório alguma característica peculiar que permite a sua identificação? Fodor (1983) argumentou que os sentidos são input systems que possuem certas características que, no conjunto, qualificam o sistema como modular: ele pode recepcionar um domínio específico de informações; tem operações internas rápidas, isoladas do restante da cognição e fora de controle voluntário; possui contrapartida definida na arquitetura neuronal; gera outputs relativamente econômicos, isto é, não-conceituais (Fodor, 1983, p. 47-101). Assim, sob o prisma de Fodor, a modularidade é a característica distintiva dos sentidos. Outra tradição, que remonta a Kant, afirma que a receptividade - "a possibilidade de sermos afetados pelos objetos" (Kant, CRP, A26/B42) - é a característica distintiva do sistema sensório.

Para Prinz, modularidade e receptividade podem ser combinadas.

The receptivity proposal is right to say that the senses serve the function of responding to inputs. The modularity proposal is right to say that the senses serve functions that are domain-specific. If we put these ideas together, the senses can be regarded as systems that respond to particular classes of inputs. This does not mean the senses are passive or impenetrable. Instead, it means that each retains a crucial degree of independence, processing its own preferred stimuli in its own preferred way. The idea can be summarized by saying that the senses are dedicated input systems (Prinz, 2002, p. 115).

Essa passagem é muito significativa. Primeiro: sentidos são systems. Segundo: são input systems. Terceiro: são dedicated input systems. Acerca do primeiro ponto: uma modalidade sensorial é um sistema pois é dotada de operações e representações próprias, que têm lugar em certa rede neuronal. Quer dizer: no plano físico, uma modalidade sensorial se distingue das demais pela contraparte neuronal. "To say that senses are systems means that they can be divided up internally, in our case, by distinct collections of cooperative neural populations" (Prinz, 2002, p. 116). Em última instância, essa afirmação é baseada em estudos recentes que usam neuroimagem funcional.

Quanto ao segundo ponto: uma modalidade sensorial é um sistema de entrada (input system), pois recebe informações do exterior do cérebro. Como representacionista, Prinz se contrapõe à tradição da embodied cognition. Aqui, especificamente, isso significa que uma modalidade sensorial não se distingue fisicamente pelo(s) órgão(s) a ela associado e tampouco pelos receptores, que podem estar em diversas partes do corpo. Consequentemente, informações do exterior do cérebro podem ser oriundas tanto do ambiente externo quanto de outras partes do corpo. 
Por fim, uma modalidade sensorial é um sistema de entrada dedicado a certa classe de informações. "[...] different senses are specially tuned to different kinds of physical magnitudes. For example, vision responds to wavelenghts of light, audition responds to frequency of molecular motion, and smell responds to molecular shapes" (Prinz, 2002, p. 117). A partir de uma classe de informações, assim interpreto, dada modalidade sensorial produz representações perceptuais de certo tipo. "Neurons in the visual system represent things such as lines or shapes, but it is able to do so by responding to patterns of light" (p. 117).

Ora, se cada modalidade sensorial é um sistema dedicado a certo tipo de informação oriunda do exterior e se as modalidades sensoriais produzem representações perceptuais de diferentes tipos, então a mente humana resulta ser algo comparável a uma central multimídia. Haveria de endossar essa analogia quem afirma que "each modality furnishes the mind with a multitude of representations turned to various aspects of the environment" (Prinz, 2002, p. 143).

Vimos, brevemente, o que são representações perceptuais e como elas se formam. Segundo o autor da proxytype theory, essas representações (i) podem ser armazenadas na memória de longa duração; (ii) depois de armazenadas elas podem ser modificadas, tanto em termos de refinamento quanto de acréscimo de informações; (iii) elas podem ser agrupadas em coleções. "For example, imagine seeing a gorilla that produces a representation that is similar, but not identical, to a stored gorilla representation. After this encounter, the new representation may be stored together with the old" (Prinz, 2002, p. 145).

Representações agrupadas em coleções formam o que Prinz chama long-term memory networks. "Long-term memory networks can come to store various kinds of information about commonly encountered categories" (p. 146). Este exemplo ilustra o desenvolvimento de uma long-term network:

For example, a gorilla might beat its chest. Because the same object was observed both standing still and beating its chest, the perceptual representations corresponding to these two states get grouped together. [...] If I hear a sound of gorilla beats its chest, I may store a record of the sound along with the visual representation of arm movements (Prinz, 2002, p. 145).

Cumpre enfatizar que, via de regra, uma long-term network vai crescendo em informações ou, se preferir, tornando-se gradativamente mais densa. Considere, por exemplo, a sua long-term network acerca de gorilas: Você sabia que o DNA desses animais é $95 \%$ igual ao dos humanos? Você sabia que gorilas são capazes de demonstrar compaixão por humanos e por outros primatas? Você sabia que o tempo de gestação de um gorila também é 
de nove meses? E que gorilas recém nascidos pesam, em média, cerca de $2 \mathrm{~kg}$, mas crescem duas vezes mais rápidos do que bebês humanos? Se alguma dessas informações é nova, então a sua long-term network acerca de gorilas acaba de tornar-se mais densa.

Em síntese, a proxytype theory considera que a nossa memória de longa duração guarda redes de representações perceptuais mais ou menos densas. Isso posto, podemos avançar para a próxima questão: Qual é a relação dessas redes com os conceitos? A resposta, já anunciada acima, é que conceitos são cópias de representações perceptuais. Desdobrarei esse entendimento na próxima seção.

\section{Copias de representações perceptuais}

Segundo Prinz, "concepts are copies or combinations of copies of perceptual representations" (2002, p. 108). A justa compreensão dessa ideia de cópia demanda distinguir memória operacional de memória de longa duração. Em uma frase: o que está na memória de longa duração (isto é, em uma longterm memory network) pode ser ativado na memória operacional (também conhecida como 'memória de trabalho'). Vejamos.

Primeiro: a memória operacional não possui a mesma capacidade da memória de longa duração. Logo, não é possível ativar - no sentido de trazer para a memória operacional - todas as informações de uma long-term network. Ativa-se um ou outro aspecto, por meio de algum conceito. Nas palavras de Prinz: "concepts are mental representations of categories that are or can be activated in working memory. I call these representations 'proxytypes', because they stand in as proxies for the categories they represent" (Prinz, 2002, p. 149).

As representações perceptuais, densas em informações e organizadas em redes, estão na memória de longa duração. Podemos ativar um ou outro aspecto dessa rede de informações. Ativar significa exatamente trazer para a memória operacional, o que é possível mediante proxytypes. Nesse processo, conceitos têm lugar. Por isso, Prinz defende que conceitos são proxies: na memória operacional, um conceito está para certa rede de representações perceptuais.

Conceitos são proxytypes. Um proxytype representa, na memória operacional, um aspecto - um subconjunto preciso de informações - de uma rede maior de representações perceptuais guardada na memória de longa duração. Por isso, Prinz chega a dizer que conceitos são representações derivadas (2002, p. 237).

Por que não identificar o conceito com a totalidade de informações da long-term memory? Essa sugestão vem da psicologia cognitiva. De acordo com 
Machery (2009, p. 12), "within psychology, the theoretical term 'concept' is commonly used as follows: a concept of $x$ is a body of knowledge about $x$ stored in long-term memory and that is used by default in the processes underlying most, if not all, higher cognitive competences when these processes result in judgments about $x$ ". Prinz, no entanto, não pensa assim.

Concepts cannot be identified with the totality of category knowledge stored in long-term memory because it is difficult to determine where knowledge of one category begins and that of another category ends. [...] Different concepts can encode some of the same knowledge (2002, p. 148).

A proxytype theory considera que o campo dos conceitos é a memória operacional. Claro está, também, que conceitos e representações perceptuais não são o mesmo. Aqueles são uma derivação destas. Proxies, em uma palavra. Desse modo, a teoria de Prinz acomoda elegantemente uma afirmação recorrente segundo a qual os conceitos são os constituintes dos pensamentos. Com efeito, um pensamento ocorrente é algo que tem lugar, por assim dizer, na memória operacional. Quando penso no meu cachorro, estou ativando na memória operacional um subconjunto de informações da extensa long-term network relacionada ao cachorro. Qual subconjunto? Depende da circunstância. "Every long-term-memory network of perceptual representations contains many overlapping proxytypes. Context determines what proxytype is used in working memory on any given occasion" (Prinz, 2002, p. 149).

Proxytypes são, por natureza, modais. Para Prinz, não faz sentido depositar esperanças em uma concepção amodal. Esse aspecto da teoria é melhor detalhado em The return of concept empiricism (Prinz, 2005, p. 686): "Concepts are not couched in an amodal code. Their features are visual, auditory, olfactory, motoric, and so on". Segundo o autor, pesquisas de Barsalou, Damásio e Lakoff, dentre outros, fundamentam essa posição.

Vimos, até aqui, que conceitos são cópias de representações perceptuais. Além desse papel, de trazer para o pensamento informações já memorizadas, os proxytypes de Prinz cumprem outra função: eles são mecanismos de detecção. Com eles, o indivíduo detecta entidades no mundo. Esse é o assunto da próxima seção.

\section{Proxytypes são indicadores e detectores}

Para a proxytype theory, conceitos cumprem dois papéis principais: constituintes do pensamento e detectores. Prinz lança mão do binômio indicator-detector para detalhar sua compreensão do vínculo da linguagem com 
o mundo: "An indicator is an unstructured entity that falls under the nomological control of some property. A detector is a mechanism that mediates the relation between an indicator and the property it indicates. Both indicators and detectors carry information" (Prinz, 2002, p. 123-124).

O binômio indicator-detector pode funcionar no âmbito de um realismo forte. É preciso que o mundo, ele mesmo, seja estruturado. Esse é um pressuposto importante da proxytype theory. Nas palavras do autor (2002, p. 06): "I assume that my FROG concept really refers to a naturally delineated category, despite the fact that I might misclassify a few instances".

Em The return of concept empiricism (2005) esse papel de detectores é apresentado por meio de uma comparação com o racionalismo de Fodor:

Fodor's way of setting things up distinguishes two broad functions for concepts: rationalists say that concepts are primarily in the business of representing, and opponents of rationalism say that concepts are primarily in the business of doing. This distinction should not be regarded as a disjoint dichotomy. Empiricists do not deny that concepts representing. Rather, they claim that concepts have other equally important functions. Empiricists say that concepts must be able to representing things in a way that facilitates interaction with those things. Representing must be in the service of doing (Prinz, 2005, p. 681).

O 'negócio do fazer' pode ser parafraseado de vários modos: interagir, manipular, organizar, categorizar... enfim, uma gama de ações que são, sob certo ângulo, cognitivas e que, justamente por isso, contribuem para "mobiliar" a mente. Nesse mesmo texto, Prinz sintetiza sua teoria empirista da mente nos seguintes termos:

\begin{abstract}
At first, creatures were input-output machines. The world cause sensory stimulation, and sensory stimulation caused programmed responses. [...] Over time, creatures evolved the capacity to store perceptual records of objects that they had encountered in the past, as well as the past consequences of those encounters. This allowed for much creative flexibility of response. Such creatures can respond differently to different objects even if they were not hardwired to recognize those objects. Finally, creatures evolved the capacity to reactivate the stored perceptual records in the absence of sensory stimulation, and they capacity to manipulate those records in working memory (Prinz, 2005, p. 688).
\end{abstract}

Ao invés de discutir implicações e desdobramentos dessa visão, gostaria de propor (ensaio meu) um esquema que procura retratar o núcleo da proxytype theory. Os números indicam o que a seguir será chamado 'Passo 1', 'Passo 2' e assim por diante: 


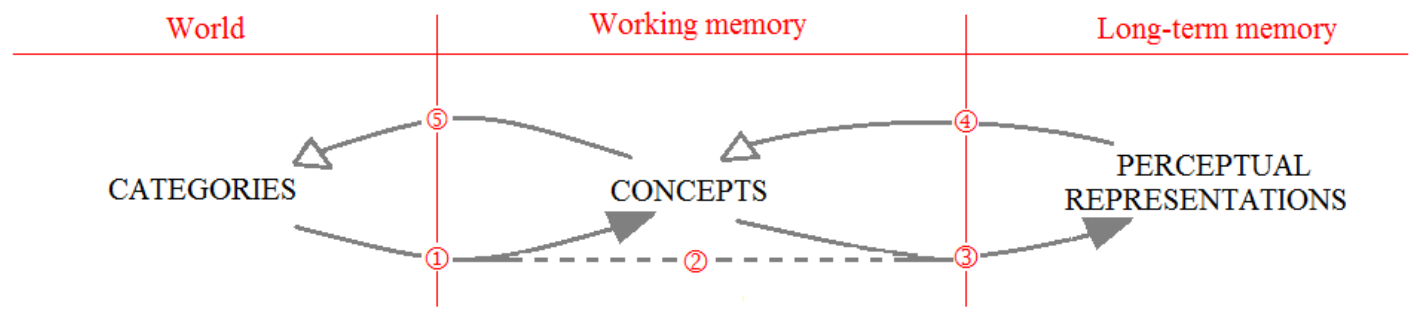

Temos, como ponto de partida, um mundo estruturado em categorias (realismo forte). Graças ao seu sistema sensorial, seres humanos percebem o mundo (passo 1), podendo memorizar as representações perceptuais (passo 3). Ao afirmar que nem todas as representações perceptuais são conscientes, Prinz está assumindo a existência de representações não conceituais (passo 2). De modo bem esquemático, é assim que os seres humanos "mobiliam" suas mentes. Os seres humanos possuem capacidade de pensar, o que ocorre quando informações de representações perceptuais são ativadas na memória operacional (passo 4). Finalmente, uma mente "mobiliada" lida melhor com o mundo, pois os conceitos cumprem também o papel de detectores (passo 5).

A theory oferece uma visão bem articulada da natureza dos conceitos, de como eles se formam e operam. Trata-se, sem dúvida, de um trabalho de fôlego, que procura guardar sintonia com outras áreas do conhecimento, especialmente as ciências cognitivas e as neurociências. "Proxytype theory is a hybrid, but its many facets can be unified under a single overarching idea: concepts are mechanisms that allow us to enter into perceptually mediated, intentionality-conferring, causal relations with categories in the world" (Prinz, 2002, p. 164).

Concluo, assim, o primeiro intento desse trabalho: oferecer uma visão geral da teoria e destacar que Prinz vincula, mas não identifica, conceitos com representações. Na próxima parte, focarei o tratamento que a proxytype theory confere à intencionalidade.

\section{O conteúdo intencional}

Em filosofia, costuma-se dizer que conceitos possuem intencionalidade, significando com isso que eles cumprem uma função referencial. Há um amplo consenso acerca desse ponto. Sintonizado com a tradição, Prinz articula as expressões 'conteúdo' e 'estado intencional': "To say that concepts have intentionality is to say that they refer, and those things to which they refer, I call 
their intentional contents. Intentional states can refer to both actual things and merely possible things" (2002, p. 03-04).

Como o conteúdo intencional de um proxytype é determinado? De acordo com Prinz, um proxytype refere os objetos ou propriedades que são capazes de causá-lo de maneira confiável. No que segue, procuro detalhar essa resposta. Para tanto, considero útil oferecer um panorama rápido das diversas teorias filosóficas da intencionalidade e, em seguida, mostrar que Prinz endossa certa versão da teoria informacional.

\section{Teorias filosóficas da intencionalidade: um panorama}

Embora todos concordem que conceitos possuem intencionalidade, há pouco consenso quando se trata de explicar como eles obtêm seu conteúdo intencional. Com efeito, há pelo menos quatro perspectivas teóricas envolvidas nessa disputa no terreno da intencionalidade: as teorias informacionais, as teorias da semelhança, as teorias descritivistas e as teorias causais. Prinz, veremos em seguida, prefere a perspectiva informacional e, por isso, dirige críticas às outras três.

As teorias da semelhança concordam que a referência se dá basicamente por semelhança, no sentido imagético dessa palavra. Por conta disso, Prinz as critica em termos de imprecisão e insuficiência. Ao prever que um conceito é como uma imagem e que tudo o que é semelhante a essa imagem cai sob esse conceito, torna-se difícil distinguir 'cachorro' de 'lobo', por exemplo. Eis a imprecisão. Ademais, há conceitos cuja representação dificilmente pode ser uma imagem, como é o caso de 'verdade' ou 'virtude'. Eis a insuficiência.

Estou de acordo com essas críticas de Prinz. Não obstante, ao menos no campo da geometria essa perspectiva cumpre o seu papel no âmbito da intencionalidade: um triângulo é um triângulo em virtude da semelhança com triângulos anteriormente conhecidos. O mesmo vale para as demais figuras geométricas planas e espaciais.

As teorias descritivistas - Prinz usa a expressão 'satisfactional theories' - dizem que algo cai sob um conceito quando satisfaz certas condições necessárias e suficientes. Prinz questiona: como essa relação de satisfação é alcançada? Como algo (uma coisa) satisfaz certo conceito? As respostas descritivistas, diz o autor, não conseguem dar conta dessa questão. Além disso, seus partidários não têm uma boa explicação para a criação de novos conceitos. Amparando-se em Kripke e Putnam - que insistiram que nós lidamos bem com certos conceitos sem conhecer as condições necessárias e suficientes -, Prinz sumariza a crítica ao descritivismo nos seguintes termos: "If 
concepts were built out of defining features, then the proposal could work, but hardly any concepts are built up of defining conditions. If concepts were built out of nondefining features, the proposal could not work, because it would assign the wrong referents to our concepts" (2002, p. 238).

As teorias causais defendem que a referência depende de uma história ou, como se lê às vezes, de um "batismo inicial": um conceito é usado pela primeira vez na presença de um exemplar da categoria que ele refere e, daquele momento em diante, as representações farão remissão a esse batismo inicial. As objeções de Prinz a essa perspectiva são: primeiro, o mencionado encadeamento ou remissão causal até o batismo inicial não é necessário para referir. Isso torna-se óbvio, ele diz, quando pensamos em conceitos de coisas fictícias/inexistentes como 'flogisto' ou 'unicórnio'. Se conceitos fossem oriundos de batismo inicial, como sugerido, jamais teríamos tais conceito em nosso repertório. Segunda objeção: um objeto particular cai sob várias categorias. Tomemos, por exemplo, o conceito 'besta' e a proposta do batismo inicial na presença de um membro dessa categoria. O caso é que essa besta também é membro da categoria 'animal', da categoria 'comida de leão', da categoria 'quadrúpede' e assim por diante. Terceira objeção: como as teorias causais explicam o fato de nós termos conceitos que referem de diferentes maneiras? "We have concepts that refer to individual objects, concepts that refer to whole kinds, and concepts that refer to appearances" (p. 240).

Creio que essa apresentação panorâmica das teorias filosóficas da intencionalidade ajuda a situar a teoria informacional da intencionalidade que Prinz recomenda. Esse é o assunto da próxima seção.

\section{Uma teoria informacional da intencionalidade}

Prinz acredita que o conteúdo intencional de um conceito é aquilo que causa o conceito como token. Locke, uma das fontes de inspiração da proxytype theory, pensava que as ideias simples referem objetos externos mediante certa covariância causal com tais objetos: "in simple ideas the mind is wholly confined to the operation of things upon it" (Locke, Essay, Book II, Ch. 30, §2, p. 267).

Nas mãos de Prinz, a noção lockeana de covariância causal ganha status de covariância nomológica. A fórmula com a qual o conteúdo intencional de um conceito pode ser especificado é a seguinte:

Xs nomologically covary with concept C when, ceteris paribus, Xs cause tokens of $\mathrm{C}$ in all proximate possible worlds where one possesses that concept. 
Vejamos a covariação nomológica através de um exemplo.

Suppose that I form the concept $W$ by encountering a wildebeest. Suppose that $W$ is reliably caused by wildebeests but not, say, dogs and turtles. Why does $W$ refer to wildebeests and not the entire class of animals or the more specific class of brindled gnus? W does not refer to the entire class of animals because animals do not cause it to be tokened in proximate words where there are no wildebeests. It does not refer to brindled gnus because, while these cause tokens of $\mathrm{W}$ in all proximate worlds, so do members of the other wildebeest subspecies. Since the class of wildebeests is constituted by all its subspecies, the fact that they all nomologically cause $W$ tokens supports the claim that $\mathrm{W}$ refers to wildebeests and not any single subspecies. Or, to put it differently, it refers to all the subspecies (Prinz, 2002, p. 242).

Salvo interpretação mais acurada, Prinz propõe uma versão da teoria causal que abre mão do regresso ao batismo inicial. Como ele mesmo diz, "a concept refers to whatever has the power cause it" (2002, p. 243). Aqui, é mais uma vez oportuno recordar que a proxytype theory encontra abrigo no realismo forte. Ela funciona na explicação do conteúdo intencional de conceitos que denotam classes tidas como naturais. Está em jogo a noção de detecção, mencionada na seção anterior.

Creio que teoria informacional da intencionalidade que Prinz propõe pode ser criticada por imprecisão. Em poucas palavras, coisas de diferentes categorias podem causar tokens do mesmo conceito. Pensemos em um sujeito que possui o conceito 'porco do mato' e, na presença de um javali, acha que este é um token de 'porco do mato'. O javali, um animal parecido com o porco do mato, causa o conceito 'porco do mato' ilicitamente. Como resolver esse problema? Como especificar o conteúdo intencional do conceito 'porco do mato' de maneira que ele não se confunda com o conteúdo do conceito 'javali'?

A fim de contornar esse problema, Dretske (1981) conjectura que existe um período inicial de aquisição e aprendizagem do conceito. Nesse período, supostamente, o conceito é aplicado com um cuidado maior. As coisas que causam o conceito durante esse período constituem seu conteúdo intencional. Uma vez aprendido, o conceito passa a ser usado de um modo mais elástico outros tipos de coisas passam a causá-lo. Prinz avalia essa sugestão e conclui que ela é equivocada. Visualizamos o equívoco quando pensamos no caso de uma criança que está aprendendo o conceito de 'pato'. Ela mal adquiriu o conceito e logo o aplica a patos, patos de brinquedo, cisnes e gansos. Se é assim, então Dretske erra ao considerar que as coisas que causam o conceito 'pato' durante o período de aprendizagem passam a estar incluídas em seu conteúdo intencional. A bem da verdade seria o contrário: no período inicial o 
conceito é mais elástico e, com o tempo, ganha precisão.

Embora discordando de Dretske, Prinz segue sua linha de raciocínio quando escreve:

I propose that the intentional content of a concept is the class of things to which the object(s) that caused the original creation of that concept belong. Like Dretske's account, mine appeals to learning, but what matters here is the actual causal history of a concept. Content is identified with those things that actually caused the first tokenings of a concept (what I call the 'incipient causes'), not what would have caused them (2002, p. 250).

Essa passagem é central para compreender a resposta de Prinz para a questão de como os conceitos obtém seu conteúdo intencional. As causas incipientes, ou seja, os primeiros exemplares percebidos, providenciam o conteúdo intencional do conceito. Isso pode ser esquematizado da seguinte forma:

$\mathrm{X}$ is the intentional content of $\mathrm{C}$ if

(1) Xs nomologically covary with tokens of $C$ and

(2) an $\mathrm{X}$ was the incipient cause of $\mathrm{C}$

A cláusula (2) chama a atenção para a importância da causa incipiente. Esse é o lado psicológico/privado/subjetivo do conceito. "Incipient causes are a special subset of actual causes. They are the causes on the basis of which a concept is formed, not just any causes that happen to occur in the history of a concept" (p. 251). Mas essa cláusula não é suficiente. Se fosse, então a teoria informacional da intencionalidade nada seria além da teoria causal. $O$ diferencial em relação à concepção causal é a cláusula (1), da covariância nomológica. "Nomological covariance delimits the set of potential intentional contents. It determines what sort of concept something is and the class of lookalikes to which it would respond" (p. 251).

The return of concept empiricism (2005) reapresenta essa teoria da intencionalidade com roupagem ligeiramente diferente:

A concept represents a category when two conditions are met:

Nomological causation: the concepts is disposed to be reliably activated by encounters with members of the category, and

Etiological causation: encounters with members of the category played a role in the acquisition of the concept (Prinz, 2005, p. 682).

Concluo, aqui, o segundo intento deste estudo: expor o tratamento que a proxytype theory confere ao conteúdo intencional. Na próxima seção, tentarei mostrar que essa concepção de intencionalidade é imprecisa. Com efeito, 
coisas de diferentes categorias podem causar tokens do mesmo conceito.

\section{As confusões da imprecisão}

Penso que a teoria da intencionalidade de Prinz é imprecisa. Coisas de categorias bastante diferentes podem causar tokens do mesmo conceito sem desrespeitar as cláusulas (1) e (2) apresentadas na seção precedente. Creio que consigo demonstrar isso por meio de um contraexemplo.

Permita-me relatar como adquiri o conceito 'Mädel' (moça, em alemão). Embora nascido no Brasil, minha língua materna é alemão. O que segue ocorreu em meados dos anos 80 , quando eu tinha cerca de 4 anos de idade e vivia em uma propriedade rural dos meus avós paternos.

Um vizinho, com quem tínhamos pouco contato, comprou um carro novo: um Fusca (Käfer na Alemanha, Beetle nos Estados Unidos, Fusca no Brasil). Vi esse carro pela primeira vez enquanto caminhava com minha avó. $\mathrm{O}$ carro passou e minha avó disse: "Hmm... es Mädel, wie schön". O termo 'Mädel' era novidade pra mim, assim como o carro. No meu entendimento, estabeleceu-se que 'Mädel' referia aquele modelo específico de veículo. Essa é a circunstância na qual adquiri o conceito, por assim dizer. Ora, nas semanas subsequentes eu vi o carro com alguma regularidade, sempre conduzido pela filha do vizinho, a motorista da família. Quando o carro passava, meus pais ou minha avó comentavam a novidade com frases como "Das Mädel kommt noch mal", "Das Mädel fährt fast jeden tag in die Stadt", "Das Mädel fährt zu schnell... heute is es durch geflogen". Todos esses usos do termo 'Mädel' referiam a moça, em seu desempenho como condutora do veículo. Para mim, no entanto, todos os comentários confirmavam que 'Mädel' designava realmente o Fusca. Considero que esse foi o período de aprendizagem do conceito.

Depois de certo tempo, passei a usar o termo 'Mädel' nas interações linguísticas com meus familiares, em declarações tais como "Ich habe es Mädel gesehn", "Hier in der Stadt gibt es mehrere Mädels", "Das Mädel gefällt mir",... Os retornos eram positivos, isto é, meus interlocutores não notaram, em momento algum, que estávamos falando de coisas diferentes. Assim, tornei-me um usuário competente desse termo singular.

Voltemos, agora, às cláusulas de Prinz:

$X$ is the intentional content of $C$ if

(1) Xs nomologically covary with tokens of $C$ and

(2) an $X$ was the incipient cause of $C$

Aplicando-o ao caso relatado temos: 
Fusca é o conteúdo intencional de Mädel se

(1) Fuscas covariam nomologicamente com tokens de Mädel e

(2) Um Fusca foi a causa incipiente de Mädel.

O caso envolve usuários competentes de uma linguagem. Há comunicação e entendimentos acerca de coisas e eventos no mundo. Não obstante, por um longo período, usamos 'Mädel' para designar coisas muito diferentes.

\section{Considerações finais}

Filósofos, psicólogos e estudiosos de outras áreas do conhecimento têm, nas ultimas décadas, formulado diversas teorias relativas à natureza dos conceitos e das representações. $O$ presente trabalho debruçou-se sobre a proxytype theory de Prinz. Segundo essa teoria, conceitos são cópias de representações perceptuais que, na memória operacional, cumprem duas importantes funções: pensar e interagir com o mundo.

Além de oferecer uma visão geral da proxytype theory, mostrei que ela serve-se de uma teoria informacional da intencionalidade para explicar a dimensão referencial dos conceitos. Prinz entende que o conteúdo intencional de um conceito se determina mediante atenção simultânea à causa incipiente $e$ à covariância nomológica. Grosso modo, a causa incipiente nos remete a aquisição e aprendizagem do conceito enquanto a covariância nomológica aponta para o funcionamento público desse mesmo conceito.

Considero que a teoria informacional da intencionalidade que Prinz recomenda é imprecisa. Essa afirmação baseia-se no contraexemplo apresentado na última seção do texto. O caso mostra que coisas de categorias bastante diferentes podem causar tokens do mesmo conceito sem desrespeitar as cláusulas que supostamente fazem funcionar a mencionada teoria da intencionalidade.

\section{Referências}

DRETSKE, F. Knowledge and the flow of information. Cambridge: MIT Press, 1981.

FODOR, J. The modularity of mind. Cambridge: MIT Press, 1983. 
KANT, I. Crítica da razão pura. 5.ed. Tradução de Manuela dos Santos e Alexandre Morujão. Lisboa: Fundação Calouste Gulbenkian, 2001.

MACHERY, E. Doing without concepts. Oxford: Oxford University Press, 2009.

LOCKE, J. An essay concerning human understanding. 27.ed. London: Thomas Tegg and Son, 1836.

ROSA, R. Prinz's problematic proxytypes. The Philosophical Quartely, v. 55, n. 221, p. 594-606, 2005.

MARGOLIS E.; LAURENCE, S. Concepts. In: ZALTA, E. (Ed.) The Stanford Encyclopedia of Philosophy, 2012. Acessivel em: <plato.stanford.edu/archives/fall2012/entries/concepts>

PRINZ, J. Furnishing the mind: concepts and their perceptual basis. Cambridge: MIT Press, 2002.

PRINZ, J. The return of concept empiricism. In: COHEN, H.; LEFEBVRE, C. (Eds.) Handbook of categorization in cognitive science. Amsterdam: Elsevier Science, 2005. p. 679-695. 